\title{
Implementing a Canadian Shared-Care ADHD Program in Beijing: Barriers and Facilitators To Consider Prior To Start-Up.
}

\section{Sayna Bahraini}

Children's Hospital of Eastern Ontario

Alexander Maisonneuve

Children's Hospital of Eastern Ontario

Yirong Liu

Peking University Sixth Hospital

André Samson

University of Ottawa

Qian Ying

Peking University Sixth Hospital

Fei Li

XinHua Hospital

Li Yang

Peking University Sixth Hospital

Philippe Robaey ( $\nabla$ probaey@cheo.on.ca )

Children's Hospital of Eastern Ontario

\section{Research Article}

Keywords: ADHD, Shared mental health care, Barriers and Facilitators, Grounded Theory, Context

Posted Date: July 7th, 2021

DOI: https://doi.org/10.21203/rs.3.rs-420636/v2

License: (1) (1) This work is licensed under a Creative Commons Attribution 4.0 International License. Read Full License 


\section{Abstract \\ Background}

The ADHD Shared Care Pathways is a program that has been developed in Canada with two main strategies: (a) to implement shared care between general practitioners (GPs) and specialists, and (b) to implement stepped care in which the patient is treated at the most appropriate level of care, depending on complexity or outcome of their illness. The current study aims to identify challenges and facilitators in implementing this program in a Chinese context.

\section{Methods}

Two focus groups were conducted using semi-structured interviews with a total of 7 healthcare providers in Beijing. A grounded theory approach using open, axial and selective coding provided three main themes pertaining to the barriers and facilitators faced at: (1) a Social-level from of the perspectives of patients and healthcare providers; (2) at a structural-level related to both internal and external organizational environments; (3) and at the intervention-level.

\section{Results}

Results reveal multilayered challenges in implementing an ADHD Shared Care Pathways program for children in China.

\section{Conclusion}

Our study highlights the importance of consultation in a new implementation context in order to get a "lay of the land". By extension, our results demonstrate areas for service development and further research.

\section{Introduction}

Attention Deficit Hyperactivity Disorder (ADHD) is one of the most common chronic childhood neurodevelopmental disorders with an estimated prevalence of 5.7 percent of children in China (1). ADHD is characterized by developmentally inappropriate inattention, hyperactivity and/or impulsiveness, and has a high persistence into adulthood (2). This disorder is often associated with academic underachievement (3), emotional liability (4), and behavioural problems (5). The relative mortality rate for this condition is surprisingly high $(2.1$ [95\% $\mathrm{Cl}: 1.7-2.5])$, with accidents being the most common cause of death (6). ADHD that is allowed to persist into adulthood can have negative consequences in an individual's personal (difficulty with relationships and a higher risk of drug and substance abuse) and professional life (difficulty maintaining jobs, absenteeism) (7). 
Although ADHD is chronic and often comorbid, it is also treatable. There is considerable evidence to support the efficacy and effectiveness of ADHD treatments (8). Although evidence for pharmacological treatments is somewhat stronger than that for non-pharmacological approaches (9), various evidencebased ADHD guidelines $(10,11)$, including Chinese guidelines (12), endorse a multi-modal approach combining medication, education and behavioural therapy. The optimal ADHD treatment effects are dependent on the quality of care and coordination of different interventions that have been planned according to patients' needs.

In the Chinese urban setting, there are several challenges to the implementation of good-quality ADHD treatment, resulting in delayed start or follow up of treatment for many patients. In these settings, primary care is delivered through general practitioners (GPs) who mostly consider themselves not sufficiently competent to take over ADHD diagnoses and treatments of children and adolescents. One of the speculated reasons for this is that GPs only receive approximately 20 hours in courses on clinical psychiatry at a university level. Given this, patients often seek out a specialist directly without registration and referral from a GP. Patients predominantly consult specialists in upper-level hospitals. Additionally, there are less than 500 qualified specialists for nearly 200 million children in China (ratio of 1:400,000). Therefore, there is a severe shortage of specialists who must deal with both complicated and uncomplicated ADHD patients. Consequently, patients are required to wait a significant amount time to get their first or follow up visit with a specialist. Taking a high-level look at paediatric ADHD diagnosis and treatment, the vast majority of children whom have the disorder are not getting the care that they need. Additionally, those that are diagnosed and treated receive care in higher-level hospitals concentrated in urban settings.

The concept of shared mental health care is presented by the Canadian Psychiatric Association and the College of Family Physicians in Canada (2000), which emphasizes collaboration between GPs and specialists in providing appropriate patient centered treatment (8). In compliance with this concept, the research team believed practical approaches to engage GPs in the treatment plan is very important. GPs can play a crucial role in the treatment of patients with uncomplicated ADHD which enables the mental health or paediatric specialist to focus more on complicated ADHD patients e.g., those with other psychiatric issues (44\%)] (13).

The ADHD Shared Care Pathways is a program that has been developed in Canada based on the concept of shared mental health care (8). This program promotes an efficient procedure where responsibilities are shared among service providers across the continuum of care. Herein, care is provided by the right person at the right time, as needed based on the severity and complexity of the personalised needs of a paediatric patient and their family. In line with the Chinese guidelines for treating ADHD (12), this program also adopts a multimodal approach that combines educational, behavioural, and medical interventions. Adaptation and implementation of the Canadian Shared Care Pathways program to the Chinese context can be a practical approach to organizing the care for children with ADHD in China and subsequently improve the quality of the treatment for this group of patients. 
This paper is part of a large joint project between Canada and China with the goal of implementing the ADHD Shared Care Pathways program in the Chinese context. The current study aims to identify challenges and facilitators in implementing this program in Beijing, China.

\section{Materials And Methods}

\section{Sample and Study Setting}

Seven healthcare practitioners participated in two semi-structured focus group sessions (see Table 1 for demographic information). The focus group sessions were held in both the Beijing Sixth Hospital and the Hai Dian Qu Wan Shou Lu Community Health Services Center.

Table 1

demographic information of the research participants

\begin{tabular}{|c|c|c|c|c|}
\hline Qualification & $\begin{array}{l}\text { Extra } \\
\text { Training on } \\
\text { ADHD } \\
\text { Management }\end{array}$ & $\begin{array}{l}\text { Work Experience in } \\
\text { years (In } \\
\text { General/The } \\
\text { Current Care } \\
\text { Setting) }\end{array}$ & $\begin{array}{l}\text { Type of } \\
\text { Setting }\end{array}$ & Role \\
\hline $\begin{array}{l}\text { Child } \\
\text { psychiatrist }\end{array}$ & Yes & $50 / 41$ & $\begin{array}{l}\text { Tertiary } \\
\text { Hospital }\end{array}$ & $\begin{array}{l}\text { As an expert to guide the } \\
\text { whole process of ADHD } \\
\text { treatment and nursing, and } \\
\text { responsible for outpatient } \\
\text { treatment }\end{array}$ \\
\hline $\begin{array}{l}\text { Child } \\
\text { psychiatrist }\end{array}$ & Yes & $21 / 18$ & $\begin{array}{l}\text { Tertiary } \\
\text { Hospital }\end{array}$ & $\begin{array}{l}\text { As an expert to guide the } \\
\text { whole process of ADHD } \\
\text { treatment and nursing, and } \\
\text { responsible for outpatient } \\
\text { treatment }\end{array}$ \\
\hline $\begin{array}{l}\text { Child } \\
\text { psychiatrist }\end{array}$ & Yes & $18 / 15$ & $\begin{array}{l}\text { Tertiary } \\
\text { Hospital }\end{array}$ & $\begin{array}{l}\text { As an expert to guide the } \\
\text { whole process of ADHD } \\
\text { treatment and nursing, and } \\
\text { responsible for outpatient } \\
\text { treatment }\end{array}$ \\
\hline $\begin{array}{l}\text { Child } \\
\text { psychiatrist }\end{array}$ & No & $32 / 10$ & $\begin{array}{l}\text { Secondary } \\
\text { Hospital }\end{array}$ & $\begin{array}{l}\text { Clinical treatment } \\
\text { (assessment and consultation } \\
\text { of ADHD pupils) }\end{array}$ \\
\hline $\begin{array}{l}\text { Director of } \\
\text { psychiatric } \\
\text { hospital }\end{array}$ & No & $32 / 5$ & $\begin{array}{l}\text { Secondary } \\
\text { Hospital }\end{array}$ & Management coordination \\
\hline Psychiatrist & No & $24 / 13$ & $\begin{array}{l}\text { Community } \\
\text { Center }\end{array}$ & Clinical treatment \\
\hline $\begin{array}{l}\text { Director of } \\
\text { Community } \\
\text { Center }\end{array}$ & No & $11 / 3$ & $\begin{array}{l}\text { Community } \\
\text { Center }\end{array}$ & Management coordination \\
\hline
\end{tabular}




\section{Data Collection}

Semi-structured focus groups were used to collect rich, qualitative data for this research. Semi-structured interviews afford interviewers the flexibility to probe particularly salient themes with participants. The effectiveness of semi-structured interviews is also greatly enhanced when used with concurrent data analysis and collection, which allows for initial interviews to inform the organization of subsequent interviews. Focus groups were done in-person and were digitally recorded (audio recording only) and transcribed as a means of ensuring accurate records and analyses.

\section{Data Analysis}

Data from the focus groups was analyzed using a Grounded Theory methodology (14). Each focus group was coded via open, axial, and selective coding, which saturated categories, identified emerging conditions, and illuminated categories of main interest (15). Data analysis was completed using the NVivo qualitative software package (version 12).

\section{Data Integrity}

Several measures were utilized by the researcher to improve credibility and trustworthiness of the analysis, including: continuous comparative analysis, immersion in the topic of study, and persistent observation of the participants (16). Dependability was adhered to through the consistent execution of study procedures. Furthermore, transferability was increased through the use of engaging in rich data descriptions, thorough questioning of the study participants, in addition to coding and analysis (16). To ensure methodological rigor, two coders independently analyzed the data and came of a consensus on themes.

\section{Results}

Several themes emerged from the data analysis of the interviews. These themes included: (1) Social-level barriers and facilitators from the perspectives of clients and healthcare provider; (2) structural-level barriers and facilitators related to both internal and external organizational environments; and (3) intervention-related barriers and facilitators. The following sections describe these themes in detail and substantiate them using in-depth quotes taken verbatim from the participants within the study.

\section{SOCIAL-LEVEL BARRIERS AND FACILITATORS}

Items were coded as social-level if they assessed barriers/facilitators that represent the social context in which the organization is located. This theme has been emerged from two aspects of clients and healthcare providers who implement the ADHD program. In the following, we will first explain barriers and facilitators on behalf of clients and then, barriers and facilitators on behalf of healthcare providers. 


\section{1-1 Social-level barriers on behalf of clients}

Parents of children with ADHD seem to harbour a lack of acceptance or distrust in the quality of General Practitioners [GPs] working within basic-level hospitals when seeking help in treating their child's mental health challenges. This lack of acceptance exists even when physicians within these hospitals are wholly qualified and trained to treat such health challenges. Instead, parents often actively seek the help of specialists within third-level hospitals. As one of the participants said: "..., if basic doctors [GPS] are trained, they may gain some knowledge about ADHD... But the question is whether the patient would like to see them...". In addition, several participants indicated that there exists an overall lack of "parents' education" on the importance of the psychological health of their children. This parental lack of education translates to a lack of screening for mental health disorders in child populations as a whole. Finally, from a logistical standpoint, participants identified geographic location and/or "traveling" as a real barrier to healthcare access. Some patients have to travel a long distance to see a specialist which is not "convenient" and "feasible" for them. Whereas if the patients' treatment was stable, they would rather to "choose a place close to home to get the medication". The long line-ups often required of patients seeking healthcare treatment was similarly seen as a barrier to specialist access: "...there lat the basic level hospitals] is no need [for patients] to queue up [compare to a third level hospital]...".

Overall, with regards to social-level barriers that pertain to the Chinese clients in the implementation of our study, lack of acceptance or distrust in the quality of physicians working at the basic-level hospital, a lack of parental education, and burdensome logistical barriers were chiefly identified.

\section{1-2 Social-Level facilitators on behalf of clients}

In addition to the social-level barriers identified by participants within the focus groups, several key facilitators were discussed to enable implementation of this project.

To begin, participants indicated that the mandate and goals of the project are highly in line with the social demands within a Chinese healthcare context which facilitates the overall acceptability of the project. Given that in the previous section, travel time and long wait times were an identified barrier to healthcare access, participants indicated that this project would help address this issue. Specifically, adopting a shared-care approach would promote the visiting of GPs at basic level hospitals and subsequently translate into lowered costs and faster patient turn-around. Mentioned during one of the focus groups: "It's quite easy to see a doctor in our hospital [basic level hospital]... there is a certain discount for taking medications from basic hospitals... and there is no need to queue up ... So, the patients may go there". Furthermore, this project would promote the education of parents, teachers, and classmates of children within ADHD, allowing for greater assistance for identification and management of children with ADHD. As one of the participants mentioned, "... it is necessary to provide education in this area. One is education for parents and the other is education for teachers".

Developing a working partnership between GPs, specialist, teachers, and decision makers have been mentioned recurrently as another facilitator during the focus groups. One of the research participants 
went so far as to indicate that: "The biggest highlight of this project is that to integrate different parts together. Working together to get the task done instead of fighting alone individually. Personally, I think that is the biggest highlight".

A final facilitator to social-level implementation involves the potential assessment of the acceptability of this sort of project from the patients' perspective: "This project includes some interview, and will also ask about patients' opinions, then ... modifying the referral scheme [based on patients' feedback]. Incorporating patients' feedback can increase the chance of sustainability of the project".

To conclude, the Client's social-level facilitators comprise of this project having a resonating mandate, beliefs that adopting a shared care approach would help with wait times and access to health services, promote education of parents and teachers of children with ADHD, develop a working partnership , and the ability to potentially assess of the acceptability of this project from the perspective of patients.

\section{1-3 Social-Level Barriers on behalf of healthcare providers}

Similar to social level-barriers identified by participants regarding clients, themes related to barriers faced by healthcare providers were identified. For example, in China, specialists are often the ones responsible for the training of GPs. However, given an already heavy workload and related time limitations, some of these specialists are often unable or unwilling to provide the necessary mental health training for GPs "especially when it is very likely to be a long-term thing". As one of the participants mentioned, "So, we're ...really intense with the time issue... How can I do it well, on the premise of not delaying my current work is a real challenge".

Not all physicians are allowed to prescribe stimulant medication for patients with a mental health diagnosis like ADHD. As such, participants indicated that there is a need for a streamlined "prescription certification for mental stimulants" in order to properly and succinctly treat their patients. Related to this issue is a lack of clarity with regards to legal scopes of practice. Several participants indicated that they had major concerns related to the possibility of practicing beyond their scope of practice when treating patients with mental health challenges. For example: "...For general practitioners, it is indeed a problem to practice beyond the scope, as a "specialist". So, in this part, I think we need to get it approved legally".

Finally, the qualifications of physicians from hospital to hospital, and from specialty to specialty are very different. For example, a basic level hospital may only consist of "undergraduate" and GPs while a third level hospital may house several specialists: "The qualifications of doctors at different levels are quite different... some doctors at the basic hospitals are undergraduate, some are even not". As such, an additional barrier in order to implement the project is the heterogeneity of practitioners; physicians that often come from differing backgrounds elicit a wide range of training needs in order to properly diagnose and manage patients. This situation makes the training needs of physicians in differing hospitals' levels complex. 
In summary, the social-level barriers experienced by healthcare providers that could hinder implementation encompass issues related to training such as time constrain and heavy workload of specialists, barriers to proper prescription certifications, and the varying qualifications held by physicians within differing hospitals which provoke a wide range of training needs.

\section{1-4 Social-level facilitators on behalf of healthcare providers}

The facilitators pertaining to healthcare providers in the implementation of this project were discussed at length by participants. In general, the physicians participated within the focus groups had a high degree of confidence and interest in the success of the project. For example, a participant indicated that: "I give a score of 9 [out of 10 for success of the project].../ can even give 9.5, based on history, and the scientific nature of the project, as well as social needs". Similarly, the compatibility of the project was seen to be highly in line with participants' own values and goals which can facilitate the successful implementation and high sustainability of the project. Participants often indicated that they had expectations and believed that the project would greatly "make improvement of medical treatment" within a Chinese context which is in line with the healthcare providers' own goals and values.

\section{STRUCTURAL-LEVEL BARRIERS AND FACILITATORS}

Item were coded as structural level if they assess barriers/facilitators that represent the organizations in which the new ADHD program is being implemented, and legal entity other than organizations where this program is being implemented. In the following, first the internal organization barriers and facilitators and then, the external organization barriers and facilitators have been explained.

\section{2-1 Internal organizational barriers}

Spoken of at length, participants identified several key barriers that they felt related to the internal organization of their respective hospitals that could affect the implementation of the ADHD Shared Care Pathways program within a Chinese context. Both general practitioners and specialists indicated that in order for this project to be successful, barriers related to lack of support on behalf of leaders at hospitals from all levels would need to be addressed. For example, participants indicated that a lack of internal policies that would ultimately support this new program could serve as a barrier to implementation: "... and, we also need their support... Whether the leaders of these hospitals support this kind of work also matters, which need communication and coordination".

On a related note, a lack of financial and human resources (technical and administrative staff) would greatly hinder implementation: "we should have the resources from our hospital... I don't know what the situation of the basic hospital is... Do they have enough personnel? ... To ensure we can do our work well, we must have policies and financial expenditures."

Finally, participants indicated that a lack of a proper referral system, that would refer patients from primary to specialized hospitals, was a definite barrier to project implementation: "The current referral system is still very imperfect... the problem is that the patients in Third Grade hospital are not transferred 
here [the basic level hospital]...". Therefore, there is a need to design a proper referral system between hospitals where the project will be implemented.

To conclude, internal organizational barriers identified by participants in both focus groups included a lack of organizational support, lack of financial and human resources, and an unclear or lack of an appropriate patient referral system.

\section{2-2 Internal organizational facilitators}

The compatibility of the project was seen as a major facilitator given the organizational culture present within many of the participating hospitals: "According to the history, it [the designated hospital's culture and values] is matched [with the current project]". One of the participants explained that the culture of their hospital supports innovation, and that's the reason for this compatibility. As was elaborated by another participants: "... I think there should be no problem in the aspect of culture... In fact, in terms of our values and culture, we serve the general population and definitely can provide such services for children."

Some-of the Chinese hospitals that were represented by the focus group participants have ongoing connections within their communities, including their local school systems which increase the chance of success in implementation of the project. Such connections were indicated by almost all participants: "In fact, I think, especially in psychiatry, we have done some services in schools, including primary and secondary schools, and we also have certain communication with schools."

\section{2-3 External organizational barriers}

External organizational barriers were identified in the form of lack of support on behalf of municipal and state governments. Namely, participants indicated that a lack of government-level policies that would ultimately enable the implementation of this project (and others like it) was a definite barrier:

"Some of the slogans we have been spoken for 10 years did not solve the problem, and some of the medical staff have changed careers... That's why l emphasized that in this process, we should let the decision makers [at the governmental level] participate and let them have a look at the actual situation. Otherwise, doctors in the top hospitals are too burdened to think or study at all".

\section{2-4 External organizational facilitators}

Many external organization facilitators were discussed by the participants from both focus group locations. For example, participants indicated that the mandate of the project was highly compatible with the needs of current Chinese "medical triage" practices and the current "direction of medical development", particularly in Beijing. Similarly, the project was seen to be highly compatible with the "management system of the Haidian district". 
Participants named a hospital in Beijing that has been relatively successful in the grading diagnosis, and treatment of patients by expert medical teams: :"I know they have expert teams ...such as for depression, $A D H D$, etc...the number of outpatients of their attending doctors is relatively large... Top experts have to be recommended level by level...". Since a proper patient referral system was identified as a barrier to the treatment of patients within the designated hospitals in the current study, the use of the aforementioned hospital's patient referral model was suggested as a possible facilitator in this project.

\section{INTERVENTION-RELATED BARRIERS AND FACILITATORS}

Item were coded as intervention-level if they assess barriers/facilitators that represent aspects of the proposed ADHD Shared Care Pathways program that will be implemented. In the following, first the barriers and then facilitators related to this theme have been explained.

\section{3-1 Intervention-related barriers}

The participants indicated the need for a detailed outline of the proposed program. Herein, participants wanted details on all internal procedures, especially as they pertain to the referral system. As highlighted by one of the participants: "For example, this kid has been treated, but he has relapse later. Under this situation... should we tell the child to go to the specialist directly, or wait for the specialist to come over?". Another participants mentioned: "But we should discuss what you [GPs] come to us [Specialist][to receive training] for, and what tasks you [GPS] need to solve after you go back [to your hospital]".

Related to this aforementioned barrier, participants indicated that the program outline should consider the reality or context of China's current healthcare setting. For example, this program should take into account the lack of specialists available who can provide training for GPs: "I'm also thinking about the time issue if I'm going to do something really detailed. Because there are so many things in hand...".

The final barrier applies to the current design of the training phase of the program. One of the participants feel that online training may not be sufficient for their needs: "In the past, there was such [online] training because of the inconvenient transportation. But the effect of the training was not very good". The lack of additional in-person training and/or online exams could hinder the training of those involved in the project.

Overall, intervention-related barriers encompassed a lack of a detailed program outline, a lack of specialists who can provide training, and the possibility that current training approach may be inadequate.

\section{3-2 Intervention-related facilitators}

A significant number of facilitators that were identified within this study applied to the intervention. To begin, participants described the scientific and rigorous design of the project very acceptable for stakeholders in China. As was elucidated: "the design of this research is very rigorous...there are very 
rigorous qualitative research methods... Through such a model, once established, it must be very scientific, effective and generalizable".

Related to one of the above-mentioned barriers, participants indicated that a series of exams, designed for the online course, would increase the overall effectiveness of the training: "We can add in exams. So online training plus exams, some tasks and so on. This will be better". Further related to the training aspect of this intervention, a proper amount of time allocated to specialists to prepare the courses (in the cases that they train GPs) would greatly contribute to the effectiveness of training provided by them, and dividing the training tasks among differing specialists would reduce the overall burden associated with meeting training goals.

Another suggested facilitator to the project would be ensuring that the assessment questionnaires are short and/or simple and that the project is completed in small, adaptive parts; much like a pilot: "But I still have some ideas. Don't do too much at one time, because I think starting from one point, then after you have accumulated enough experience and made some improvement, you can slowly move on". Having a clearly stated end-goal for the project would also contribute to the sustainability and overall longevity of the project.

In conclusion, the intervention-related facilitators that were discussed by participants included the strong scientific foundations that make up this study, the allocation of adequate time to prepare training, the potential of proper training assessment tools, ensuring that the questionnaires/assessments used during the study do not overwhelm participants, and having a clearly-stated end goal for the project.

\section{Discussion}

The purpose of this study was to assess the challenges and facilitators in implementing a Canadian ADHD Shared Care Pathways program in a Chinese context, specifically in Beijing. In this study, we engaged general practitioners, paediatricians, and mental health specialists who work at designated hospitals where the project will be implemented in future. Understanding the challenges and facilitators from the viewpoint of individuals who are likely to be able to use the results of this research in their practice can enhance research uptake and project sustainability in similar context (17).

\section{Laying the foundation: The healthcare landscape in China}

As western researchers, we are used to looking at ADHD treatment through a western lens. In order to reach the objectives of this research, it was important to take into account the characteristics of the Chinese healthcare system, becoming more knowledgeable about the system in which we would be working. Indeed, following economic reforms, the healthcare system in China has gone through several significant changes. Moving away from the rural Community Medical System and urban company-owned hospitals, China now has a leveled system which is managed by the Ministry of Health (18). Today, a three-tiered system is used wherein each increasing tier is dependent on the severity of a patient's illness. The first tier of care, Level 1, refers to local hospitals that use basic equipment and offer basic care. 
District, municipal, or provincial hospitals, referred to as Level 2 hospitals, have more sophisticated equipment when compared to the former level. Finally, ministry-owned central hospitals, or Level 3 hospitals, offer a wide range of specialized care, having the best available equipment in China. These hospitals are comparable to university or research hospitals in North America (18). Similar to the wide disparity in level of care based on type of hospital, there is a wide range in the level of education held by healthcare providers in China. Currently, three levels of education are available with each level dictating where a physician may practice. Those holding a primary education level (1-3 years) are able to work as "village doctors" (18). Individuals with secondary education (2-3 years) are able to work at Level 1 and Level 2 hospitals. Physicians holding tertiary education (3-11 years) work in Level 2 or Level 3 hospitals (19). The ability to prescribe drugs (and subsequently the level of care a patient receives) depends heavily on what level of education a doctor holds. Some physicians need additional training to meet requirements for the hospital level in which they are currently employed.

Identified barriers and facilitators in this study can, at their roots, be attributed to the differing sociopolitical and cultural contexts unique to the Chinese healthcare system. Although both Canada (the country that the program was originally developed in) and China have relatively strong and responsive healthcare systems, their core makeup can be very different. It is important to understand the healthcare landscape in China in order to properly situate our research within its greater context. In the current study we identified three main themes: (1) Social-level barriers and facilitators faced by healthcare users and healthcare providers; (2) structural-level barriers and facilitators related to both internal and external organizational environments; and (3) barriers and facilitators specifically related to our intervention. Understandably, these themes are a result of the differences between Occidental and Oriental approaches and therefore can both hinder or facilitate the implementation of a Canadian shared-care approach to ADHD management in a Chinese context.

\section{Socio-cultural Barriers}

As a preface, our analysis of the socio-cultural barriers in implementing a Canadian shared-care approach to ADHD treatment in China included parental lack of education and a lack of trust in the Chinese primary healthcare system, the geographic layout of Beijing hospitals, and the perceived competence of healthcare practitioners. These barriers may represent why ADHD comes to attention in some children and not in others in a timely manner. Parent's lack of education pertaining to mental health issues was one of the major socio-cultural barriers that were identified by our research participants. They believed that this, in turn, translated to a lack of treatment-seeking behaviours in patients in Beijing. This issue is exacerbated by the patients' lack of trust in the quality of Chinese GPs and their preference to visit specialists for most of their hospital needs. Given the geographic layout of Beijing, many patients must travel long distances to visit these specialists who are available in a few third-level hospitals. The cumulative effect of this ultimately results in longer wait times as more and more patients opt for this route. On the other hand, our research participants mentioned that many GPs in China do not consider themselves competent enough to take charge of diagnosis and treatment of children with ADHD, therefore, they refer the potential patients to the third level hospitals which worsen the waiting time to 
access specialists. Participants believed, varying qualifications of general practitioners in China can explain this perceived lack of competency as not all receive the same amount of medical education and not all hold prescription authorization for mental stimulants.

Clients' treatment seeking behaviours is very important for timely recognition and quality management of ADHD (20). Results of our study attribute this to parent's knowledge about mental health issues and trust in health services provided by GPs at the first and second level hospitals. Such findings bring to light the need for community education regarding indicators, proper treatment, and available healthcare services for ADHD. To date, the source of parents' lack of trust has not been studied in China, however, it can be rooted in physicians' lower level of education at primary and secondary hospitals (18). According to the China Health Statistics (18), 55.2\% of licensed general practitioners in cities and $82.4 \%$ in rural areas did not have a bachelor's degree from a medical university. There may be an expectation from patients that physicians are able to treat every illness that is presented, and that an over-expectation in physician ability may cause mistrust when is not fulfilled. Lack of parent's trust in health services has also been reported in western countries as a barrier to ADHD treatment seeking behavior (21), however, it has been attributed to other reasons. For example, Saul (2014) attributes it to parental disclosure to negative information about ADHD from non-healthcare providers or social media (22). Some African American parents also have been shown to perceive ADHD as a means to create business and enhance profits for private medical sectors $(23,24)$. Research in western countries also shows primary healthcare providers perceived incompetency to take charge of the medications of children who first have been regulated by specialists results in more referrals to specialists and longer waiting lists for patients (25). As no study has been found to report the average waiting time to visit a specialist in China or any other countries, we cannot have a meticulous comparison of this variable, however, the population of China and specialists to patient ratio of 1:400,000 suggest a critical situation for ADHD patients there.

\section{Socio-cultural facilitators}

Our research participants believed adopting the Shared Care Pathways program would help overcoming some of the aforementioned socio-cultural barriers. Using this program, GPs will be trained to provide screening and initial ADHD treatment. Training GPs not only increase their confidence in managing ADHD, but also enhances parents' trust in the services that GPs provide. This promotes patients' access to health services and initiation of timely treatment as GPs are more accessible than specialists. However, if the level of care of one patient steps up to a level above routine care, the GP is able to receive consultation form specialist or refer patients to them for advanced treatment. Participants of our research also mentioned this program will facilitate community education by providing training programs for parents and teachers about ADHD through schools. On the other hand, they believed the Shared Care Pathways program is compatible with healthcare providers' own values and goals; therefore, there would be a great interest by them to implement the program at their settings. According to the Consolidated Framework for Implementing Research (CFIR) (26), effective implementation can be anticipated by the extent to which the project goal matched to patients' and stakeholders' needs and values. The more they perceive alignment between the meaning they attach to the new approach and their needs have a strong 
impact on their acceptance of the proposed approach and subsequently the success of the implementation (26).

\section{Structural-level barriers}

Our analysis revealed several organizational barriers that can hinder the implementation of a shared-care approach to ADHD treatment in China. From a hospital-specific standpoint, lack of support from leaders, resources, and an appropriate patient referral system were identified as chief concerns. An overarching and exacerbating component could be lie in a lack of support from municipal and state governments with regards to mental healthcare in China. Therefore generally, our research participants believed for implementation of the new approach there is a need for authorization of internal and governmental policies, allocation of resources, and technical support to establish effective referral systems within the hospitals.

Leadership engagement in terms of commitment and interest in a new approach leads to a stronger implementation climate and subsequently implementation effectiveness $(27,28)$. High and middle-level leaders in the hospital play a crucial role by providing internal polices, negotiating for resources, creating a climate to support employees' learning, and fostering collaboration between different teams $(29,30)$. According to Gershon et al (2004) compatibility of the new intervention with the organization goal as well as engaging leaders in decision making process about the implementation increase the likelihood of their support (30). The capacity of an organization is also another structural factor that has a positive relationship with the implementation effectiveness. This factor includes the human, financial, technical, and physical resources necessary for an organization to provide a quality service that can be supported both at the hospital and governmental level $(31,32)$. In the long run, supportive national policy and guidelines are necessary to establish all the essential resources to improve the efficiency and sustainability of implementations (26). To date, no study in China has discussed the willingness of the government and capacity of hospitals in terms of integrating a shared-care treatment approach in mental health, specifically ADHD.

\section{Structural-level facilitators}

Our research participants believed compatibility of the project with the designated hospitals' culture (e.g., supporting new and innovative approaches) and current direction of local government can facilitate negotiation with them to overcoming structural barriers. In addition, in 2016, one of the designated hospitals (Peking University Sixth Hospital) signed an agreement with the Haidian Mental Health Hospital, and the associated community hospitals to develop a partnership to improve mental health service management in lower level hospitals. According to this agreement, these hospitals will work together to provide logistic support (e.g., space, salary, and training) and referral policies between primary and specialized services. The share care pathways program fits well with this agreement as it promotes collaborative approach between different level hospitals. According to our research participants, the designated hospitals also have a good connection with schools which facilitate the implementation of the school component of the program where collaboration between schools and hospitals will be made to 
promote ADHD screening and treatment. Cosmopolitanism is an important factor that impact the success of implementation (26). Organizations that establish external networking are more prone to implement new practices quickly (33). Peer pressure (i.e., competition between organizations) is another phenomenon that happens in highly cosmopolitan organizations which has a positive association with the success of the implementation.

\section{Intervention-level barriers}

Although high-level themes arose that pertained to patients and practitioners as well as organizational settings, participants also discussed intervention-specific barriers and facilitators. To begin, a barrier identified by participants included the lack of a detailed outline of the proposed program, especially as it pertains to the referral system and role of different levels of physicians. Another barrier to implementation identified by a research participant was the potential that our project does not take into consideration the reality of the Chinese healthcare system insofar as it relates to the amount of available specialists at each hospital. A final barrier that was identified by participants was the potential that online training sessions would be inadequate in instilling the skills and knowledge needed to successfully implement such an intervention.

Generally speaking, two main factors emerged in this section that should be given considerable attention: the issue of accountability regarding the specific context, and developing competency in stakeholders. In this context, accountability means having all levels of employees responsible for accomplishing designated tasks (34). Establishing this concept in detail (i.e., who/what/when) based on available resources and an organisation's culture has been identified as extremely helpful for the research uptake (35). In addition, developing competency in the form of training improves clinicians' knowledge and skills to address designated tasks (35). Training stakeholders has been recommended in previous studies (35-38) and should be evaluated using measures to ensure that trainees obtain an appropriate level of clinical capacity (39). Although the common 'gold standard' training for health care providers is in person workshops, supplemented with manuals and clinical supervision (40), a current meta-analysis conducted by Richmond and colleagues (2017) shows that online methods may be as effective as alternative methods for training clinicians for the outcomes of knowledge and clinical behaviour (41). Nevertheless, to date, we could not find any study directly comparing differing methods of training (online or in person) for healthcare providers in a specific country.

\section{Intervention level facilitator}

With regards to intervention-specific facilitators, participants mentioned that the Canadian Shared-Care Approach to ADHD treatment had a strong scientific foundation. Relatedly, participants indicated that having a clearly stated end goal was a definite facilitator to the project. Allocating sufficient time for specialists to prepare materials to train GPs and evaluating the effectiveness of training were also identified as factors which facilitate the success of the training phase of the project. A final facilitator lay in the fact that participants thought that the assessment tools that we proposed using in our intervention (questionnaires) would not overwhelm participants. 
The intervention level facilitators emerging from this study are in line with relevant implementation science literature $(26,35,42)$. According to Albert and colleagues (2007), stakeholders use information that they can trust (35). A strong scientific foundation in research which can be shown in the form of using rigorous methodology and scientific references supplied by a trusted international organization is more likely to get used (35). In addition, the style and format in which research evidence is being presented can have a significant impact on the attitude of stakeholders and their motivation to become involved (42). Three studies conducted in Australia, Canada, and Ireland which explore the factors influencing research utilisation in each country highlighted the importance of clear articulation of the research with the knowledge users (43-45). Compatible with the results of our study, these three studies identified having clear goals and providing well-structured research materials as intervention-related facilitators to success of implementation. Along with the aforementioned factors, the feasibility of innovation plays a crucial role in stakeholders' willingness for behavioural change. As outlined by the CFIR (2009), the level of resources that are dedicated to implementing a project such as time, funds, and staff reveals the degree of the feasibility of the innovation (26). The present study seems to support this finding, and at the same time indicates that this item is one of the major facilitators for the success of the current project, regarding the severe shortage of specialists in China.

\section{Conclusion}

We conducted a qualitative study to systematically examine the factors influencing the implementation of the ADHD Shared Care Pathways program in a Chinese context. We learned that there are a number of factors which could hinder or improve the uptake of the program in China, including issues relating to the program itself (intervention factors), aspects relating to clients and healthcare providers (socio-cultural factors), and the context where the knowledge will be used (structural factors). The identified barriers and facilitators contribute to adaptation of the program to fit local circumstances and eventually increase uptake and sustained use of the program. Two of the major barriers in China that may interfere with the implementation of the program are first, varying qualifications held by physicians within differing hospitals which suggest a wide range of training needs regarding ADHD management, and second, lack of appropriate patient referral systems between different levels of hospitals.

Our chosen approach allows us to slowly and dynamically implement a shared-care approach within a very different context in which it was first developed. Instead of inserting ourselves within a vastly different medical system and dictating changes, our adaptive approach allows for the proper identification and allocation of resources and fosters investment in the goals of the project. We are continuing our collaboration with the local stakeholders to overcome other identified barriers in order to enhance the possibility of successful implementation. An effective implementation improves timely and quality ADHD management in children and adolescents and could also be a model for many other similar conditions in China or other developing countries.

\section{Declarations}




\section{Ethics Approval and Consent to Participate}

All work was conducted with the approval of relevant ethics committees (Children's Hospital of Eastern Ontario REB Protocol No: 18/75X) and follows the principles of the Declaration of Helsinki. Consent was obtained from all participants prior to data collection.

\section{Consent for Publication}

Not applicable.

\section{Availability of Data}

The datasets generated during and/or analysed during the current study are available from the corresponding author on reasonable request.

\section{Competing Interests}

The authors declare that they have no competing interests.

\section{Funding}

This work was supported by the National Natural Science Foundation of China and Canadian Institutes of Health Research GAC-154987.

\section{Authors' Contributions}

SB performed the data analysis and was a major contributor in writing the manuscript. AM performed the data analysis and was a major contributor in writing the manuscript. YL performed the data analysis and was a major contributor in writing the manuscript. AS aided in the literature review and data interpretation. QY aided in the literature review and data interpretation. FL developed the protocol and aided in data interpretation. LY developed the protocol and aided in data interpretation. PR aided in the literature review and data interpretation.

\section{Acknowledgements}

The authors would like to acknowledge and thank those who participated in the focus groups.

\section{References}

1. Tong $L$, Shi H, Zang J. Prevalence of ADHD in children of China: a systematic review and meta analysis. Chin J Public Health. 2013;29(9):1279-83.

2. Agnew-Blais JC, Polanczyk GV, Danese A, Wertz J, Moffitt TE, Arseneault L. Evaluation of the Persistence, Remission, and Emergence of Attention-Deficit/Hyperactivity Disorder in Young Adulthood. JAMA Psychiatry. 2016;73(7):713-20. 
3. Biederman J, Petty CR, Dolan C, Hughes S, Mick E, Monuteaux MC, et al. The long-term longitudinal course of oppositional defiant disorder and conduct disorder in ADHD boys: findings from a controlled 10-year prospective longitudinal follow-up study. Psychol Med. 2008;38(7):1027-36.

4. Sobanski E, Banaschewski T, Asherson P, Buitelaar J, Chen W, Franke B, et al. Emotional lability in children and adolescents with attention deficit/hyperactivity disorder (ADHD): clinical correlates and familial prevalence. J Child Psychol Psychiatry. 2010;51(8):915-23.

5. Biederman J, Petty CR, Fried R, Kaiser R, Dolan CR, Schoenfeld S, et al. Educational and occupational underattainment in adults with attention-deficit/hyperactivity disorder: a controlled study. J Clin Psychiatry. 2008;69(8):1217-22.

6. Dalsgaard S, Østergaard SD, Leckman JF, Mortensen PB, Pedersen MG. Mortality in children, adolescents, and adults with attention deficit hyperactivity disorder: a nationwide cohort study. Lancet. 2015;385(9983):2190-6.

7. Biederman J, Wilens TE, Mick E, Faraone SV, Spencer T. Does attention-deficit hyperactivity disorder impact the developmental course of drug and alcohol abuse and dependence? Biol Psychiatry. 1998;44(4):269-73.

8. Kates N, Mazowita G, Lemire F, Jayabarathan A, Bland R, Selby P, et al. The evolution of collaborative mental health care in Canada: A shared vision for the future. The Canadian Journal of Psychiatry / La Revue canadienne de psychiatrie. 2011;56(5):1-10.

9. Sonuga-Barke EJ, Brandeis D, Cortese S, Daley D, Ferrin M, Holtmann M, et al. Nonpharmacological interventions for ADHD: systematic review and meta-analyses of randomized controlled trials of dietary and psychological treatments. Am J Psychiatry. 2013;170(3):275-89.

10. National Collaborating Centre for Mental H. National Institute for Health and Clinical Excellence: Guidance. Attention Deficit Hyperactivity Disorder: Diagnosis and Management of ADHD in Children, Young People and Adults. Leicester (UK): British Psychological Society (UK)

11. Copyright (c 2009, The British Psychological Society \& The Royal College of Psychiatrists.; 2009.

12. Pliszka S. Practice parameter for the assessment and treatment of children and adolescents with attention-deficit/hyperactivity disorder. J Am Acad Child Adolesc Psychiatry. 2007;46(7):894-921.

13. Zheng YL, J. Chinese Guidelines for the Prevention and Treatment of Attention Deficit Hyperactivity Disorder. 2 ed. Beijing: Chinese Medical Multimedia Press; 2015.

14. Bower P, Garralda E, Kramer T, Harrington R, Sibbald B. The treatment of child and adolescent mental health problems in primary care: a systematic review. Fam Pract. 2001;18(4):373-82.

15. Taylor BF, K. Interviewing and analysis. Qualitative Research in the Health Sciences: Methodologies, Method sand Processes. New York, NY: Routledge; 2013. p. 205-23.

16. Thériault A, Gazzola N. Therapist Feelings of Incompetence and Suboptimal Processes in Psychotherapy. Journal of Contemporary Psychotherapy. 2010;40(4):233-43.

17. DiMillo J, Samson A, Thériault A, Lowry S, Corsini L, Verma S, et al. Living with the BRCA genetic mutation: an uncertain conclusion to an unending process. Psychol Health Med. 2013;18(2):125-34. 
18. Macaulay AC, Jagosh J, Seller R, Henderson J, Cargo M, Greenhalgh T, et al. Assessing the benefits of participatory research: a rationale for a realist review. Glob Health Promot. 2011;18(2):45-8.

19. Milcent C. Healthcare Reform in China: From Violence To Digital Healthcare. Switzerland: Springer International Publishing; 2018.

20. Anand S, Fan VY, Zhang J, Zhang L, Ke Y, Dong Z, et al. China's human resources for health: quantity, quality, and distribution. Lancet. 2008;372(9651):1774-81.

21. Wright N, Moldavsky M, Schneider J, Chakrabarti I, Coates J, Daley D, et al. Practitioner Review: Pathways to care for ADHD - a systematic review of barriers and facilitators. J Child Psychol Psychiatry. 2015;56(6):598-617.

22. Hamed AM, Kauer AJ, Stevens HE. Why the Diagnosis of Attention Deficit Hyperactivity Disorder Matters. Front Psychiatry. 2015;6:168-.

23. Saul R. ADHD Does Not Exist: The Truth About Attention Deficit and Hyperactivity Disorder. 1 ed. New York, NY: Harper Wave; 2014.

24. Hervey-Jumper H, Douyon K, Franco KN. Deficits in diagnosis, treatment and continuity of care in African-American children and adolescents with ADHD. J Natl Med Assoc. 2006;98(2):233-8.

25. Ellison P. Science over cynicism. Children and Adults with Attention-Deficit Hyperactivity (CHADD).. Attention Magazine. 2003;1:27-33.

26. Janssen RM, Takach O, Nap Hill E, Enns RA. Time to endoscopy in patients with colorectal cancer: Analysis of wait times. Can J Gastroenterol Hepatol. 2015.

27. Damschroder LJ, Aron DC, Keith RE, Kirsh SR, Alexander JA, Lowery JC. Fostering implementation of health services research findings into practice: a consolidated framework for advancing implementation science. Implementation Science. 2009;4(1):50.

28. Klein KJ, Conn AB, Sorra JS. Implementing computerized technology: an organizational analysis. J Appl Psychol. 2001;86(5):811-24.

29. Lukas CV, Holmes SK, Cohen AB, Restuccia J, Cramer IE, Shwartz M, et al. Transformational change in health care systems: an organizational model. Health Care Manage Rev. 2007;32(4):309-20.

30. Leeman J, Baernholdt M, Sandelowski M. Developing a theory-based taxonomy of methods for implementing change in practice. J Adv Nurs. 2007;58(2):191-200.

31. Gershon RR, Stone PW, Bakken S, Larson E. Measurement of organizational culture and climate in healthcare. J Nurs Adm. 2004;34(1):33-40.

32. McKay VR, Dolcini MM, Catania JA. Impact of Human Resources on Implementing an EvidenceBased HIV Prevention Intervention. AIDS Behav. 2017;21(5):1394-406.

33. Flaspohler P, Duffy J, Wandersman A, Stillman L, Maras MA. Unpacking prevention capacity: an intersection of research-to-practice models and community-centered models. Am J Community Psychol. 2008;41(3-4):182-96.

34. Greenhalgh T, Robert G, Macfarlane F, Bate P, Kyriakidou O. Diffusion of innovations in service organizations: systematic review and recommendations. Milbank Q. 2004;82(4):581-629. 
35. Ferlie E. The Oxford Handbook of Health Care Management. UK: Oxford University Press; 2016.

36. Albert MA, Fretheim A, Maiga D. Factors influencing the utilization of research findings by health policy-makers in a developing country: the selection of Mali's essential medicines. Health Res Policy Syst. 2007;5:2.

37. Sauerborn R, Nitayarumphong S, Gerhardus A. Strategies to enhance the use of health systems research for health sector reform. Tropical Medicine \& International Health. 1999;4(12):827-35.

38. Chunharas Sea. Lessons in Research to Action and Policy - Case studies from seven countries. Geneva: The Council on Health Research for Development (COHRED) 2000.

39. Trostle J, Bronfman M, Langer A. How do researchers influence decision-makers? Case studies of Mexican policies. Health Policy Plan. 1999;14(2):103-14.

40. Li X, Krumholz HM, Yip W, Cheng KK, De Maeseneer J, Meng Q, et al. Quality of primary health care in China: challenges and recommendations. The Lancet. 2020;395(10239):1802-12.

41. Beidas RS, Kendall PC. Training Therapists in Evidence-Based Practice: A Critical Review of Studies From a Systems-Contextual Perspective. Clin Psychol (New York). 2010;17(1):1-30.

42. Richmond H, Copsey B, Hall AM, Davies D, Lamb SE. A systematic review and meta-analysis of online versus alternative methods for training licensed health care professionals to deliver clinical interventions. BMC Med Educ. 2017;17(1):227.

43. Buckley H, Tonmyr L, Lewig K, Jack S. Factors Influencing the Uptake of Research Evidence in Child Welfare: A Synthesis of Findings from Australia, Canada and Ireland. Child Abuse Review. 2014;23(1):5-16.

44. Jack S, Dobbins M, Tonmyr L, Dudding P, Brooks S, Kennedy B. Research evidence utilization in policy development by child welfare administrators. Child Welfare. 2010;89(4):83-100.

45. Arney F, Bromfield L, Lewig K, Holzer P. Integrating strategies for delivering evidence-informed practice. Evidence \& Policy: A Journal of Research, Debate and Practice. 2009;5:179-91.

46. Buckley H. Whelan S. Putting Research Evidence to Work: Key Issues for Research Utilisation in Irish Children's Services. Dublin: Children Acts Advisory Board 20092009. 